\title{
Convergence Hybrid Network Design for Advanced Multicast and Broadcasting Service based on Mobility Context Awareness Networks: Part I Constructing CAA Algorithm Hypotheses
}

\author{
Seungyong Shin and Byungjoo Park* \\ Department of Multimedia Engineering, Hannam University \\ 133 Ojeong-dong, Daeduk-gu, Daejeon, Korea \\ syshin09@hotmail.com,*bjpark@hnu.kr \\ *Correspondent Author: Byungjoo Park* (bjpark@hnu.kr)
}

\begin{abstract}
For Mobile IPv6 (MIPv6) which is based on host can have a problem due to signal process and the increase of loads that are performed at a mobile node during handover, Proxy Mobile IPv6 (PMIPv6) protocol which is based on network does not get involved in the signaling process related to IP mobility that the mobile terminal reduces the consumption of mobile assets. However, since multimedia streaming services that are supported by PMIPv6 network is always transmitted through Local Mobility Anchor (LMA), it has a disadvantage in that it can have an increased traffic and unneeded layover issues caused by the expansion of dual tunnel.

In this paper, the advanced multicast and broadcasting service (AMBS) optimization algorithm for data load balancing with Advanced Mobile Anchor Point (AMAP) is introduced and a handover priority Design Cross-Layer algorithm between L2 / L3 through the collection of Access Point information in accordance with the priority is defined according to whatever the network conditions offer.
\end{abstract}

Keywords: IAPP, HPMIPv6, FPMIPv6, Reverse Binding Update, Bi-Directional Tunnel

\section{Introduction}

The current information and communications services begun the ubiquitous era wherein the communication between all objects and smart devices becomes possible through the support of the expansion of information infrastructure and intelligent things. The M2M communication is emerging as a new growth engine that will lead the future social change.

The development of wireless mobile communications-based personal devices becomes the center of a major Internet service that intelligently focus on its mobility. Also, various efforts for the development of the mobile communication radio access technology in the next generation network Next Generation Network (NGN) were prioritized. To this end, the exemplary IP mobility protocol is standardized by the MIPv6 IETF NETLMM WG.

MIPv6 has disadvantages such as, packet losses and the waste of wireless link resources. Various efforts for the improvement of the handover latency was followed by various organizations through the implementation of networked-based mobility. Thus, the PMIPv6 protocol was standardized in order to address this issues. The main advantage of the PMIPv6 protocol is that it extends the Home Agent (HA) functionality in order to improve the efficiency of radio resources is enabled the support for mobility independence. However, the signaling procedures for PMIPv6 mobility management on the mobile terminal and the Mobile Access Gateway (MAG) causes packet loss and load is increased due to the frequent handover requests among access routers. This means that 
there is no significant difference in terms of handover delay and packet loss compared to the existing MIPv6 protocol. Therefore, in the PMIPv6 protocol, the Fast Handovers for Proxy Mobile IPv6 (FPMIPv6) technology in which FMIPv6 (RFC 5568) technology is applied to the PMIPv6 network has been standardized in order to recognize the network status information in advance and to reduce the packet loss due to the handover delay. FPMIPv6 is similar to FMIPv6 in that it pre-predicts handover signals in the L2 layer using the trigger information and tunnels them, but PMIPv6 handles the signal in the network instead of participating in any signalling when the terminal performs a handover. Therefore, it is possible to minimize the packet loss due to the handover delay in the data flow area. However, the FPMIPv6 technique also causes unnecessary generation of buffer space due to the handover signal prediction and thereby, packet loss still remains as a problem to be solved.

In this paper, the local mobility management via Round Trip Time (RTT) introduces the Advanced Mobility Anchor Point (AMAP) which context information for efficient transfer for packet loss minimization. The AMAP domain effect under mobility management of MAG which was utilized for Inter-Access Point Protocol (IAPP) information is available for prior collection of base stations corresponding to the MAG.

Furthermore, it minimizes the handover latency caused by the Reverse Binding Mechanism due to the route optimization procedure. In addition, it also minimizes the handover delay due to the route optimization procedure with the Reverse Binding Mechanism.

\section{Related Works and Problem}

\subsection{Mobile IPv6}

MIPv6 is a representative IP mobility protocol standardized by IETF. It is proposed in RFC3775 in order to solve the problem of service disconnection that occurs when a terminal supporting wireless network moves to a new network area and to provide seamless communication between terminals. The whole MIPv6 handover procedure is shown in Figure 1. In the operational procedure, the movement of the MN is detected through Movement Detection (MD). The movement detection is performed through an Unsolicited Router Advertisement message or a Neighbor Unreachable Detection (NUD) process that is periodically received from the New Access Router (NAR) message when the current AP (PAP) signal strength is low and packets are no longer transmitted through the currently connected router (PAR). After the movement is confirmed, the mobile station generates the CoA using the prefix information of the router, and the validity of the address is verified through the Duplicated Address Detection (DAD) process. The MN then performs a location registration procedure through a Binding Update (BU) / Binding Acknowledge (BA) message through which the MN informs the Home Agent (HA) and the Correspondent Node $(\mathrm{CN})$ of its current location. Then, the Return Routability (RR) is performed as an optional procedure in order to increase the reliability of the procedure when selective security is required during the BU process. MIPv6 is one of the reliable mobility support technologies in wireless IP network, but it has limitations due to session disconnection caused by long handover delays, waste of wireless link resources, and nonpreference of communication carriers. 


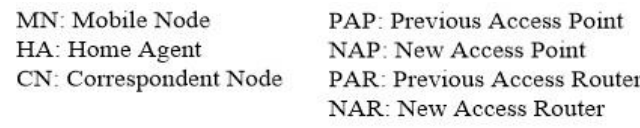

MIPv6(Mobile IPv6)

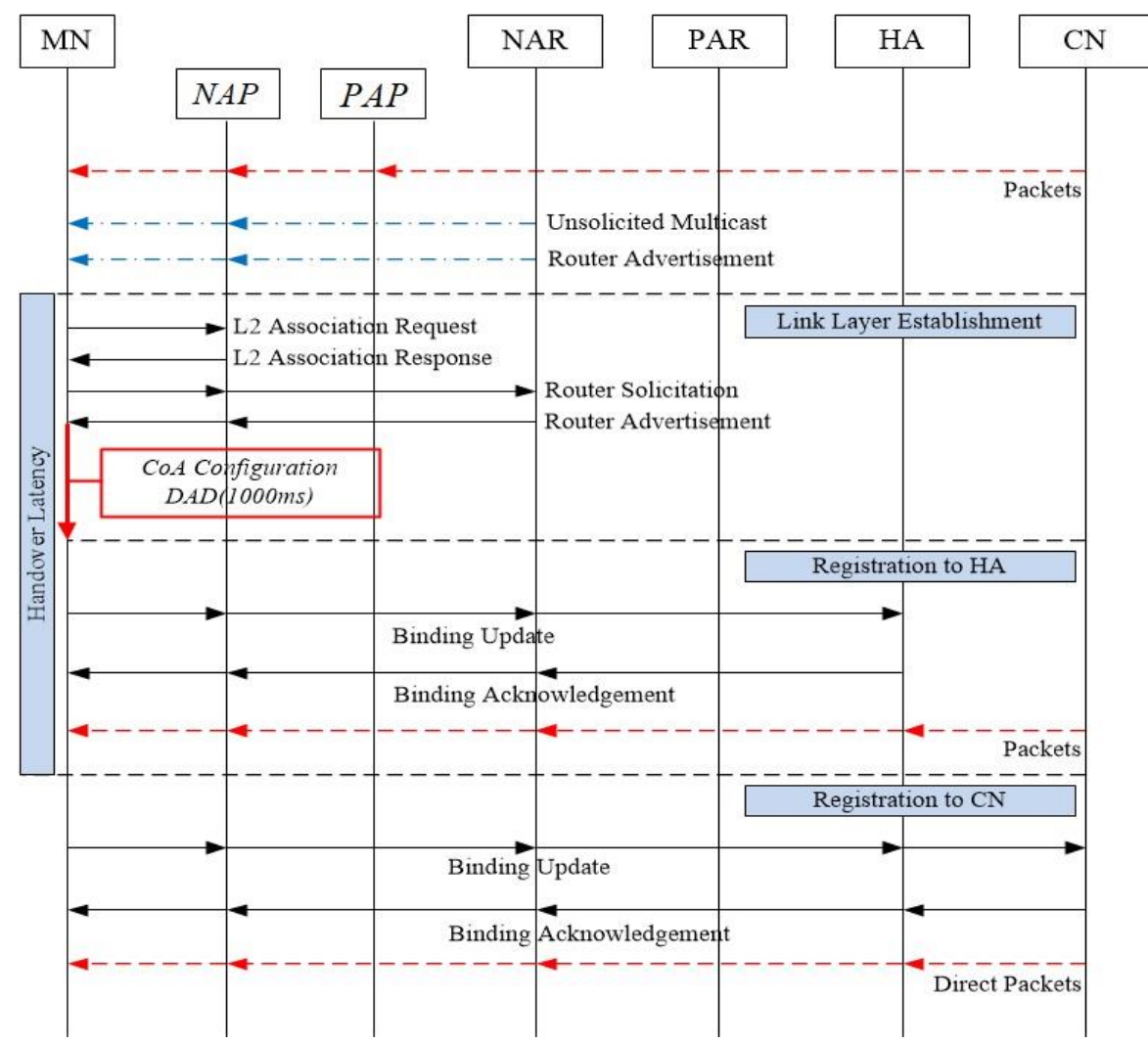

Figure 1. MIPv6 Handover Procedure

\subsection{FMIPv6(Fast Mobile IPv6)}

FMIPv6 was proposed by IETF to detect the movement of Access Points (APs) in advance in order to improve the handover performance of MIPv6, and it is now established as RFC 5568. FMIPv6 minimizes the L3 latency, which is a problem of existing handover, by detecting the movement of the terminal in advance through the L2 Trigger information and configuring the IP tunnel, thereby enabling seamless final handover. The entire FMIPv6 handover procedure is shown in Figure 2. FMIPv6 detects the handoff of the AP signal of the router that is currently receiving the service or performs handover whenever the information of the access router that is being serviced differs. At this time, the terminal confirms the PAP information of the currently connected PAR.

\subsection{FMIPv6(Fast Mobile IPv6)}

FMIPv6 was proposed by IETF to detect the movement of Access Points (APs) in advance in order to improve the handover performance of MIPv6, and it is now established as RFC 5568. FMIPv6 minimizes the L3 latency, which is a problem of existing handover, by detecting the movement of the terminal in advance through the L2 Trigger information and configuring the IP tunnel, thereby enabling seamless final handover. The entire FMIPv6 handover procedure is shown in Figure 2. FMIPv6 detects the handoff of the AP signal of the router that is currently receiving the service or performs handover whenever the information of the access router that is being serviced 
differs. At this time, the terminal confirms the PAP information of the currently connected PAR.

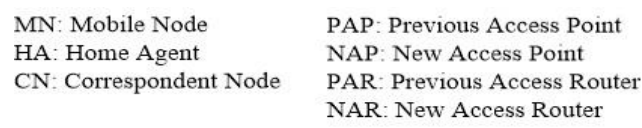

FMIPv6(Fast Handovers for Mobile IPv6)

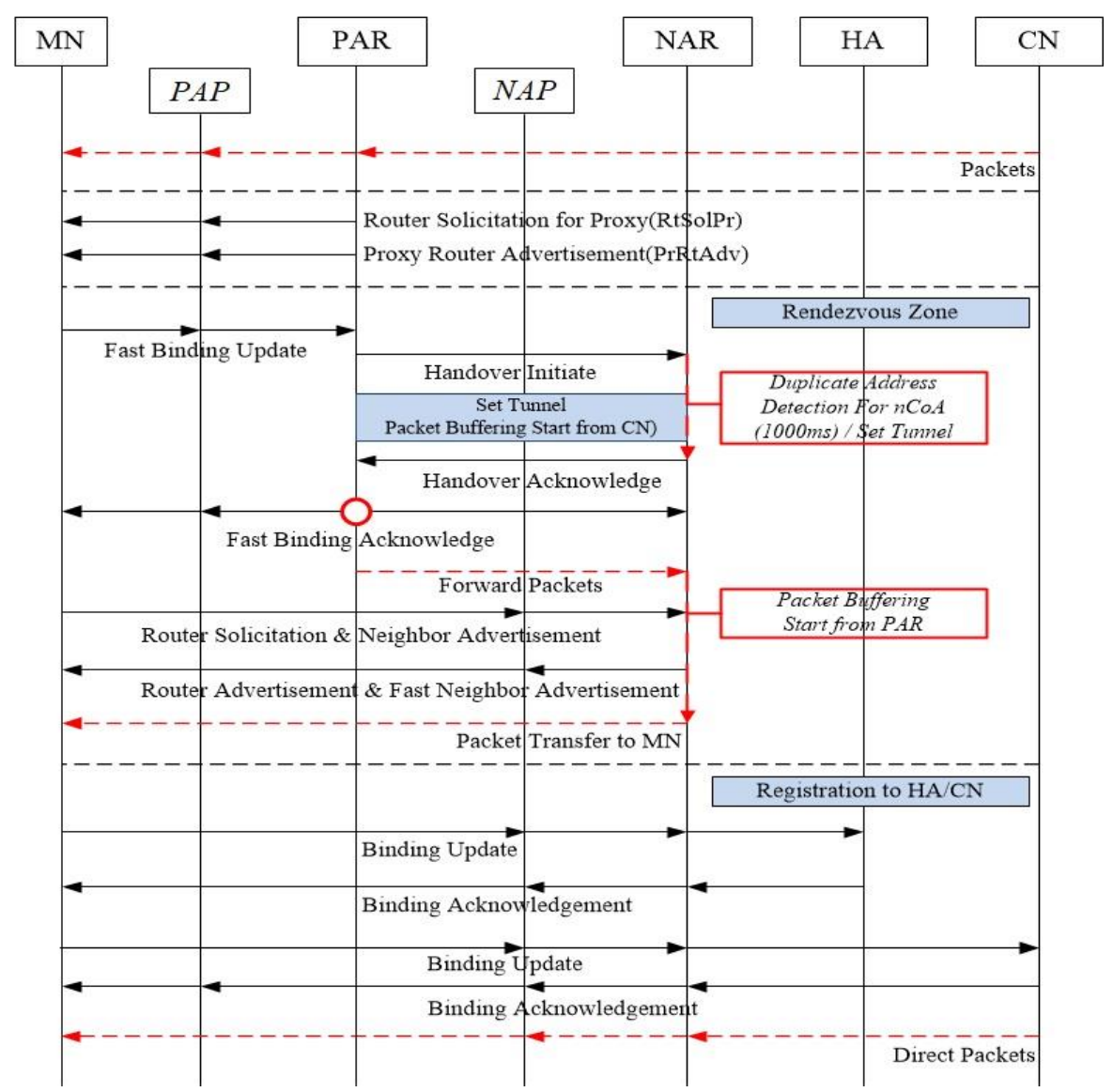

Figure 2. FMIPv6 Handover Procedure

The router sends a Router Solicitation for Proxy (RtSolPr) and receives a Proxy Router Advertisement (PrRtAdv) message in response to it. The PrRtAdv message contains the prefix information of the NAR. The MN that receives the PrRtAdv message sends a Fast Binding Update (FBU) message to the PAR. The PAR then sends a Handover Initiate (HI) / Handover Acknowledgment (HAck) message to the NAR in order to perform DAD and tunnel creation. After that, the PAR continuously monitors the result notification and the L2 trigger and sends a Fast Binding Acknowledgment (FBAck) message to the terminal and the NAR to confirm whether or not the MN is disconnected. A Fast Neighbor Advertisement (FNA) Packets tunneled from the PAR are buffered in the NAR until the $\mathrm{RS}$ / RA message containing the information is exchanged. After that, the MN transmits a $\mathrm{BU}$ message to the $\mathrm{HA}$ and the $\mathrm{CN}$ to perform the basic procedure for the location registration. When the MN receives the response, all procedures related to the FMIPv6 handover are completed. 


\section{Routing Optimization using Context-Awareness Algorithm (CAA) for HFPMIPv6 (CAA-HFPMIPv6)}

In this chapter, we explain the context awareness algorithm (CAA) based on bidirectional tunnel scheme for mobility prediction at L2 level and routing optimization at L3 level. In this paper, it is assumed that the terminals are located in overlapping AP areas of access routers under AMAP1 and AMAP2 domains in order to prove efficient management of regional mobility management by selective Mobile Access Gateway (MAG) selection in FPMIPv6 network. PMAG periodically collects the neighboring network information through IAPP for the network construction. The procedure of the proposed method is as follows. Figure 3-4 shows the CAA-HFPMIPv6 Conceptual Design Procedure and handover movement flow.

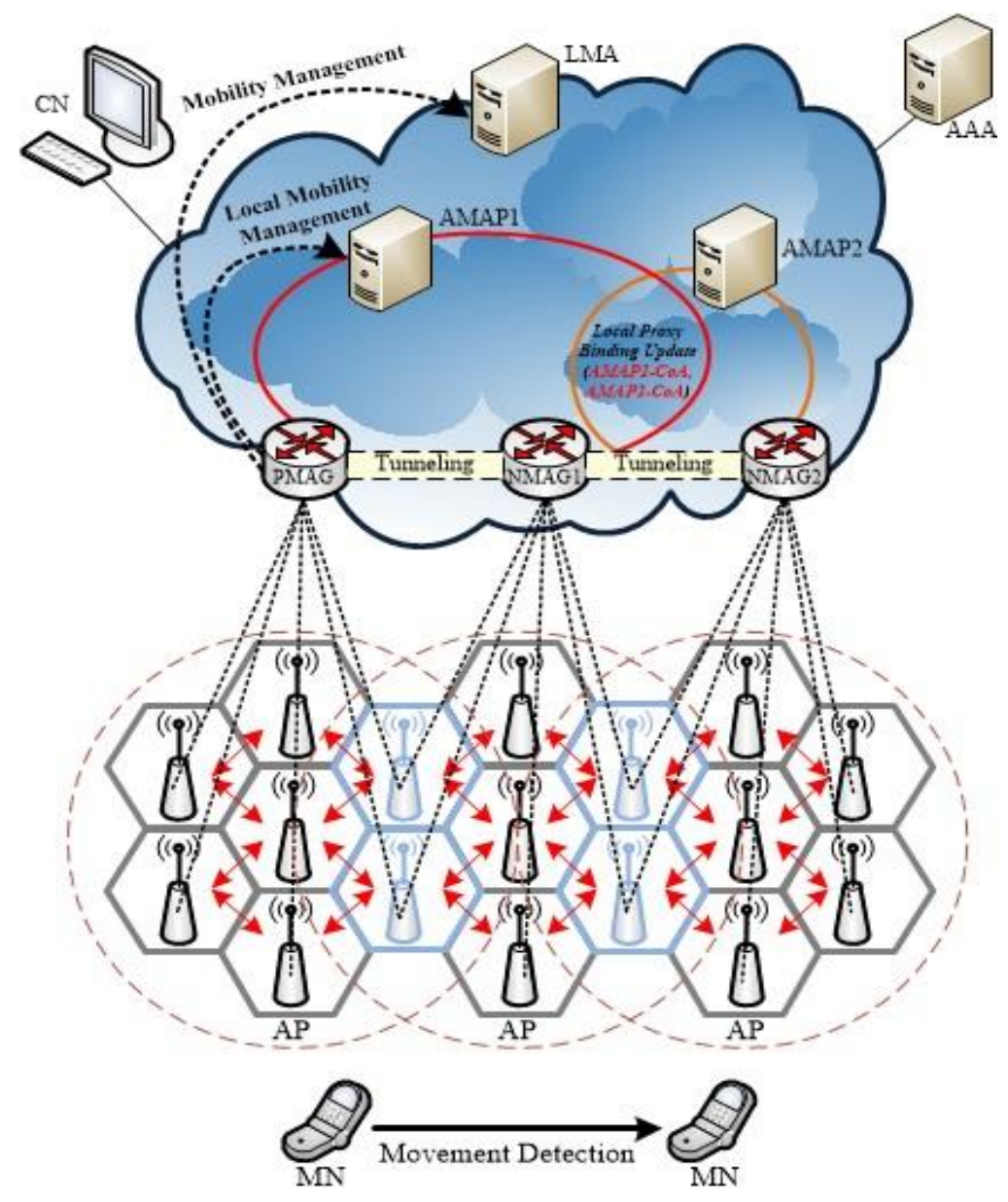

Figure 3. Conceptual Design Procedure

1) First, in order to collect the preliminary information about the overlapped network domain area, the currently connected router (PMAG) pre-collects the AP information for the overlapping Advanced Mobile Anchor Point (AMAP) domain area using IAPP and stores it in the Policy Store (PS).

2) When the $\mathrm{NAP}_{\text {power }} \geq \mathrm{PAP}_{\text {power }}$ is received through the L2 Trigger signal, the MN exchanges a message for Network Access Identifier (NAI) authentication with a Previous Mobile Access Gateway (PMAG) to receive mobility in the network area. 


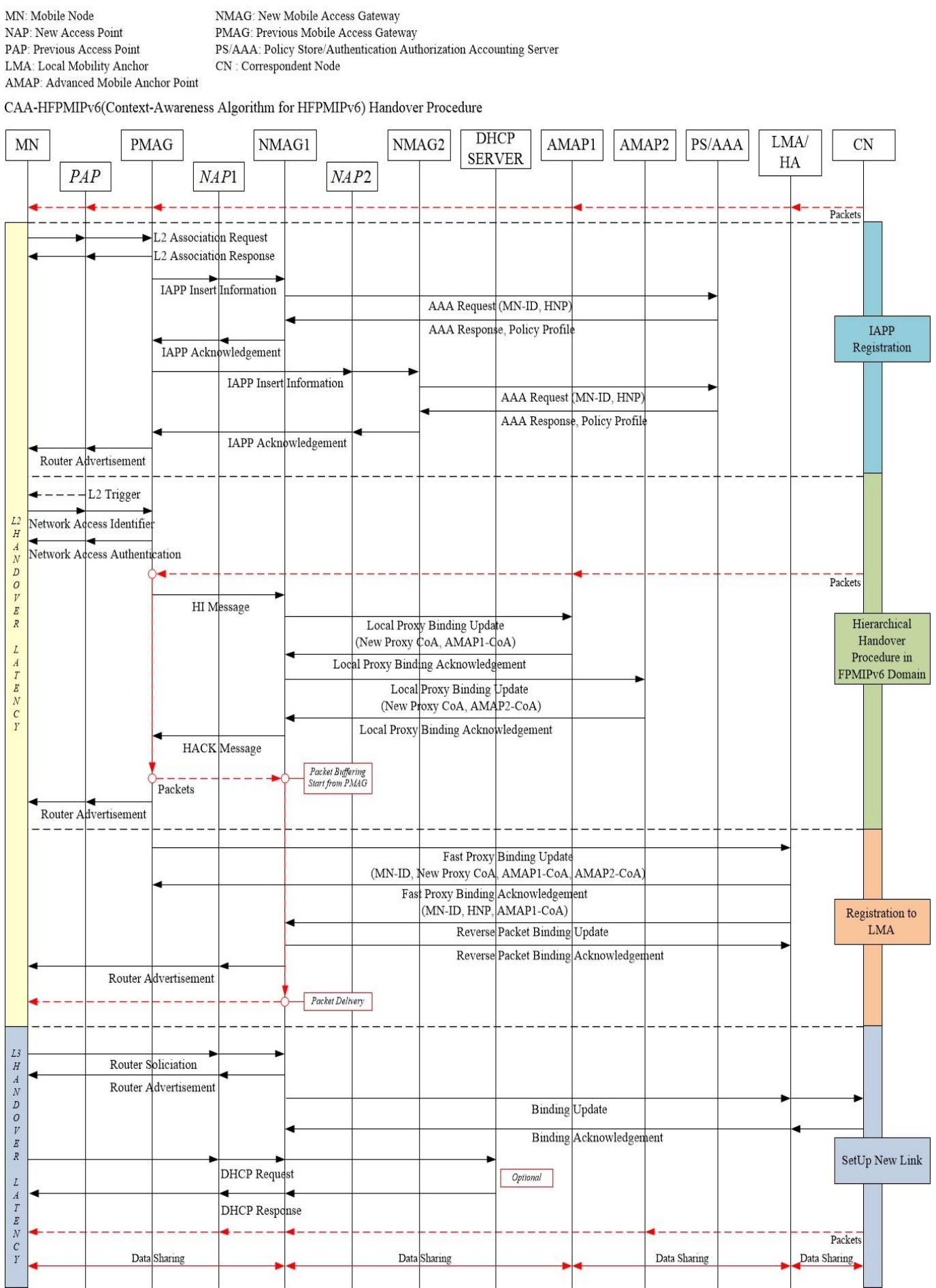

Figure 4. CAA-HFPMIPv6 Handover Procedure

3) After that, the MS exchanges Handover Initiate (HI) / Handover Acknowledgement (HACK) messages between the PMAG and the New Mobile Access Gateway (NMAG) to reduce the signaling load for efficient mobility support to form a bidirectional tunnel. The message includes MN-Identifier (MN-ID), Link Layer Identifier (LL-ID), AMAP1-CoA, AMAP2-CoA and Home Network Prefix (HNP). In this case, NMAG (NMAG1) sends a Local Proxy Binding Update (LPBU) / Local Proxy Binding Acknowledgment (LPBAU) 
message to AMAP1 and AMAP2 in order to generate and maintain a binding update list for the overlapping AMAP regions.

4) The PMAG then sends the information to the MN through a Router Advertisement (RA) message and at the same time the Local Mobility Anchor (LMA) and the Fast Proxy Binding Update (FPBU) / Fast Proxy Binding Acknowledgment (FPBAck) are used to update the location information The LMA sends and receives Reverse Proxy Binding Update (RPBU) / RPBAck (Reverse Proxy Binding Acknowledgment) messages to the router (NMAG1) to be served through the Reverse Binding Mechanism.

5) Upon completion of the Reverse Binding procedure with the LMA, the MN sends and receives a Router Advertisement (RA) message according to a Router Solicitation (RS) request to and from the NMAG1 in order to perform the Router Discovery process. The location registration procedure with the LMA / CN is performed in PMIPv6 (RFC5213). It follows the outlined basic procedures.

Table 1 depicts the HI / HACK message format. The addition to the Option field ( $\mathrm{H}$ Flag) in the HI message can determine if there is a movement within the same domain to which a temporary tunnel is created. The movement is within the AMAP domain area based on the information about the overlap region.

Table 1. HI/HACK Message Format

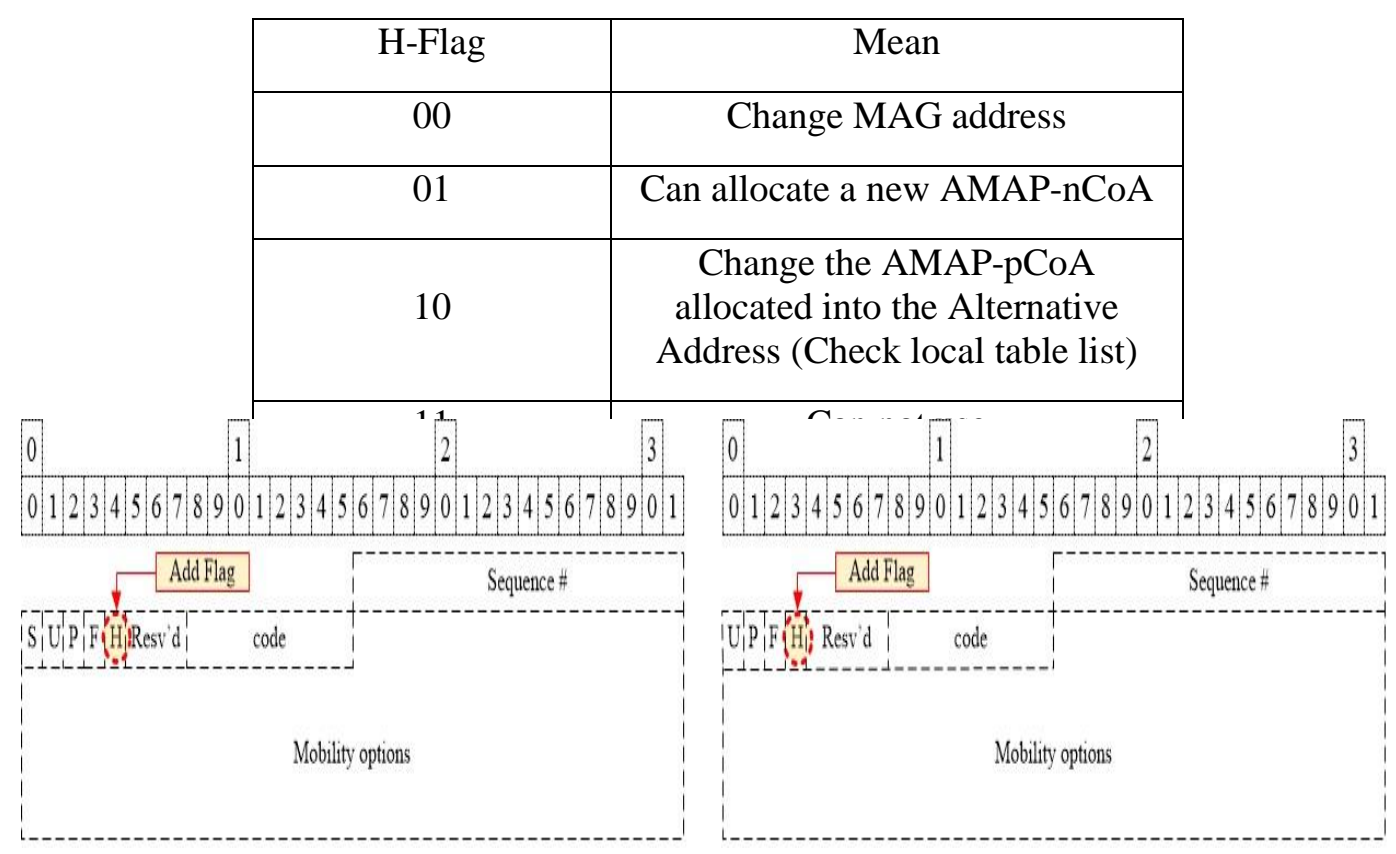

\section{Conclusion}

The network-based mobility protocol, PMIPv6 is standardized to support the reliability and efficient mobility in IETF. However, the frequent handover in the limited network region can cause packet losses due to complex signaling. This paper adopted IAPP and AMAP to support advanced high-speed handover support in Fast Proxy Mobile IPv6 which considering the network size, and it is expected to reduce the signaling load by predicting the mobility of terminal and through a Cross-Layer algorithm design between L2/L3. In addition, it reduce the handover delay time that occurs when optimizing the route using Reverse Binding mechanism, when the region that a terminal wants to transfer to is not a transfer (domain level) within cross-laying AMAP domains that is currently accessing, it can reduce the handover delay time by not performing the routing path 
optimization process, when offering the real-time multimedia data service on the wireless network in the future, Quality of Service (QoS) is expected to offer users a guaranteed service.

\section{Acknowledgements}

This research was supported by Basic Science Research Program through the National Research Foundation of Korea (NRF) funded by the Ministry of Science, ICT and future planning (2015R1A2A2A03002851).

\section{References}

[1] T. Narten, E. Nordmark, W. Simpson and H. Soliman, "Neighbor Discovery for IP version 6(IPv6)", IETF RFC 4861, (2007).

[2] N. Moore and G. Daley, "Fast address configuration strategies for the mobile internet", The Australian Telecommunications, Networks and Applications Conference (ATNAC), (2003).

[3] C. Perkins, D. Johnson and J. Arkko, "Mobility Support in IPv6", IETF RFC 6275, (2011).

[4] H. Soliman, C. Castelluccia, K. ElMalki and L. Bellier, "Hierarchical Mobile IPv6 (HMIPv6) Mobility Management", IETF RFC 5380, (2008).

[5] S. Gundavelli, K. Leung, V. Devarapalli, K. Chowdhury and B. Patil, "Proxy Mobile IPv6", IETF RFC 5213, (2008).

[6] H. Yokota, K. Chowdhury, R. Koodli, B. Patil and F. Xia, "Fast Handovers for Proxy Mobile IPv6", IETF RFC 5949, (2010).

[7] A. Shin Mishra and W. Arbaugh, "An Empirical Analysis of the IEEE 802.11 MAC Layer Handoff Process", ACM SIGCOMM Computer Comm, Rev. vol. 33, no. 2, (2003).

[8] D. Johnson, C. Perkins and J. Arkko, "Mobility Support in IPv6", IETF RFC 3775, (2004).

[9] S. Kent, "IP Encapsulating Security Payload (ESP)", IETF RFC 4303, (2005).

[10] R. Koodli, "Mobile IPv6 Fast Handovers", IETF RFC 5568, (2009).

[11] B. Park, Y.-H. Han and Haniph Latchman, "A Study on Optimal Fast Handover Scheme in Fast Handover for Mobile Ipiv6 Networks”, LNCS, vol. 4412, (2007).

[12] K. Kong, W. Lee, Y. Han, M. Shin, and H. You, "Mobility management for All-IP mobile networks:Mobile IPv6 vs Proxy Mobile IPv6", IEEE Wireless Communications, (2008).

[13] C. Makaya and S. Pierre, "An Analytical Framework for Performance Evaluation of IPv6-Based mobility Management Protocols", Wireless Communications, vol. 7, (2008), pp. 972-983.

[14] S. Park, and Y. Choi, "Performance Analysis of Hierarchical Mobile IPv6 in IP-based Cellular Networks", Proceedings of IEEE Personal, Indoor and Mobile Radio Communications, (2003), pp. 2818-2822.

[15] J.-Y. Choi, S. Yang and J. Jeong, "On QoS Provisioning Based on User Mobility Patterns for Proxy Mobile IPv6 Networks", In Computer Science and its Applications, (2015), pp. 983-989.

\section{Authors}

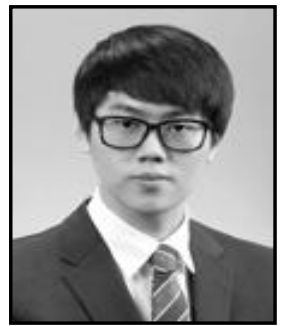

Seungyong Shin, he received the M.S. degree in Multimedia Engineering from Hannam University, Daejeon Metropolitan City, Rep. of Korea in 2015. Presently, he is on the Ph.D. Course in Multimedia Engineering at Hannam University and one of the members of Mobile and Multimedia Communication Laboratory. His research interests are Wireless Mobile Network (MIPv6, PMIPv6), IEEE 802.11f (Inter-Access Point Protocol), IEEE 802.16e (Mobile WiMAX), Wireless Sensor Network, Real-Time Multimedia Transmission Service.

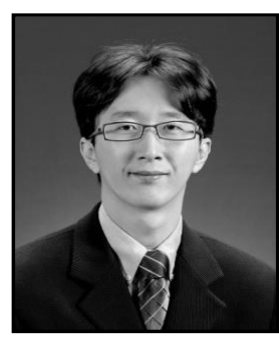

Byungjoo Park, he received the B.S. degree in electronics engineering from Yonsei University, Seoul, Rep. of Korea in 2002, and the M.S. and Ph.D. degrees (first-class honors) in electrical and computer engineering from University of Florida, Gainesville, USA, in 2004 and 2007, respectively. From June 1, 2007 to February 28, 2009, he was a senior researcher with the IP Network Research 
Department, KT Network Technology Laboratory, Rep. of Korea. Since March 2, 2009, he has been a Professor in the Department of Multimedia Engineering at Hannam University, Daejeon, Korea.He is a member of the IEEE, IEICE, IEEK, KICS, and KIISE. His primary research interests include theory and application of mobile computing, including protocol design and performance analysis in next generation wireless/mobile networks. He has published approximately 35 research papers on the theory and application of mobile computing, IPTV, Internet ApplicationSince 2004, his activities have focused on IPv6, IPv6 mobility, media independent handover, and cross-layer optimization for efficient mobility support on IEEE 802 wireless networks. He is an honor society member of Tau Beta Pi and Eta Kappa Nu. His email address is vero0625@hotmail.com and bjpark@hnu.kr. 
International Journal of Future Generation Communication and Networking Vol.10, No.8 (2017) 